\title{
A Case of Daptomycin-Induced Immune Thrombocytopenia
}

\author{
Charles Grégoire, ${ }^{a}$ Caren Brumpt, ${ }^{\text {b }}$ Delphine Loirat, ${ }^{\text {a }}$ Nicolas Lau, ${ }^{\text {a }}$ Cedric Bruel, ${ }^{\text {a }}$ Francois Philippart, ${ }^{\text {a }}$ Carine Couzigou, ${ }^{c}$ \\ Maité Garrouste-Orgeas, ${ }^{a}$ and Benoit Misset ${ }^{\mathrm{a}, \mathrm{d}}$ \\ Intensive Care Unit, Saint Joseph Hospital Network, Paris, France; ; Department of Hematology, Lariboisière Hospital, AP-HP, Paris, France ${ }^{\text {b; }}$ Antibiotic Management Team, \\ Saint Joseph Hospital Network, Paris, France; ${ }^{c}$ and René Descartes University, Paris Sorbonne Cité, Paris, France ${ }^{d}$
}

We report a case of severe daptomycin-induced immune thrombocytopenia in a patient treated for methicillin-resistant Staphylococcus epidermidis and ampicillin-resistant Enterococcus faecalis bacteremia acquired in an intensive care unit. Serum antibodies bound to platelets in the presence of daptomycin on flow cytometry. There was no evidence of other causes of thrombocytopenia. The patient died of brain herniation complicating extensive cerebral hemorrhage. To our knowledge, this is the first described case of daptomycin-induced thrombocytopenia.

W e report a case of daptomycin-induced immune thrombocytopenia observed in a 64-year-old man admitted to our intensive care unit (ICU) for septic shock due to mesenteric vein thrombosis. Surgery was performed, and empirical antibiotic therapy with ceftriaxone, metronidazole, and a single gentamicin dose was initiated. The mesenteric vein thrombosis was treated with unfractionated heparin. On day 18, a further episode of sepsis developed. Vancomycin and piperacillin-tazobactam were started because of an abdominal abscess positive for Staphylococcus epidermidis, Enterococcus faecalis, and Pseudomonas aeruginosa. On ICU day 29, i.e., 10 days after its initiation, vancomycin was stopped and replaced with daptomycin at $6 \mathrm{mg} / \mathrm{kg}$ once a day (3, 4). Piperacillin-tazobactam was followed up. Four days after daptomycin initiation, extensive cutaneous purpura developed and the platelet count dropped to less than $10 \times 10^{9} /$ liter. The profound thrombocytopenia prompted us to stop piperacillin-tazobactam, daptomycin, and heparin. No effect was seen after intravenous immunoglobulin and corticosteroid therapy. On day 5 after the platelet count drop, the patient developed a severe cerebral hemorrhage with a coma. The platelet transfusion was initiated, but hydrocephalus occurred, and 4 days later, the platelet count was normal but the patient died of brain herniation.

Investigations of the cause of the patient's thrombocytopenia were conducted. A bone marrow aspirate contained numerous megakaryocytes, indicating platelet destruction in the circulation. The patient was afebrile, and the biological sepsis markers were improved (leukocyte count, 12,700/ $\mathrm{mm}^{3}$; procalcitonin level, 0.59 $\mu \mathrm{g} /$ liter). Coagulation times were normal, and serum fibrinogen was $4.5 \mathrm{~g} /$ liter, ruling out disseminated intravascular coagulation. Results were negative from a test for anti-PF4 antibodies done to look for heparin-induced thrombocytopenia. There was no evidence of thrombotic microangiopathy (absence of hemolysis and renal or neurological failure). Flow cytometry showed daptomycin-dependent binding of antibodies to normal platelets (Fig. 1). No antibodies dependent on piperacillin-tazobactam were detected.

Regarding the role of daptomycin, several aspects of our case deserve discussion. First, the time from daptomycin initiation to the diagnosis of thrombocytopenia was 4 days. Drugdependent antiplatelet antibodies usually develop only after 1 to 2 weeks of drug exposure (1). However, the rapid drop in the patient's platelet count strongly suggests immune-mediated thrombocytopenia. Moreover, a 4-day period remains consistent with drug-induced thrombocytopenia (5). Second, many

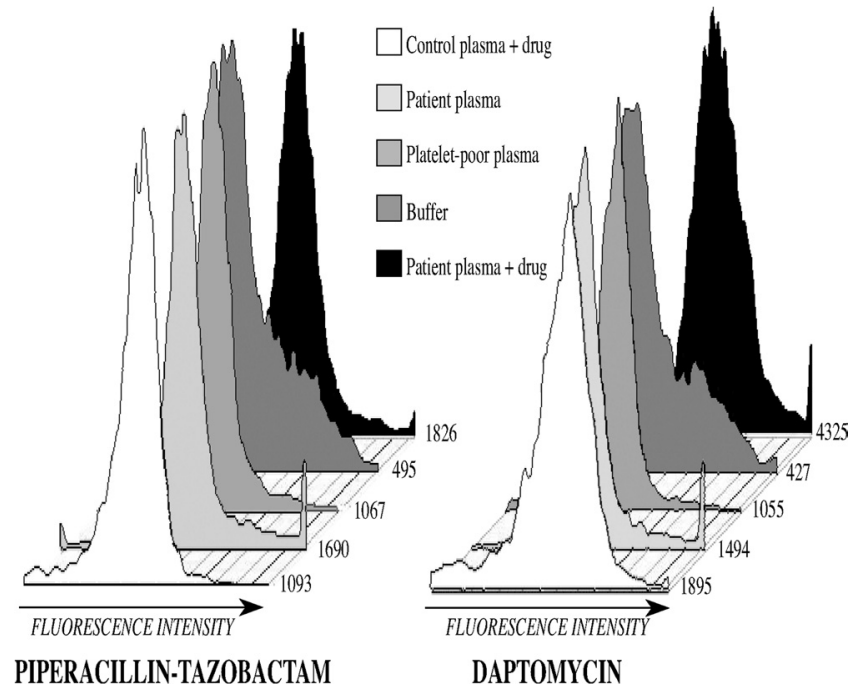

FIG 1 Flow cytometry analysis for the presence of a daptomycin-dependent, platelet-reactive antibody. The platelet median fluorescence intensity (MFI) was significantly higher with the patient's serum plus daptomycin (MFI, 4,325) than with the patient's serum plus buffer (MFI, 1,494) or normal serum plus daptomycin (MFI, 1,829). No such difference occurred with piperacillin-tazobactam (left).

factors capable of inducing thrombocytopenia may occur in critically ill patients. Among these factors, the most common were ruled out. Furthermore, in the presence of daptomycin, flow cytometry showed elevated platelet surface-bound immunoglobulins and serum antiplatelet antibodies, indicating immunological platelet destruction. Although a role for daptomycin is probable, the exact mechanism underlying the patient's thrombocytopenia remains unclear. Drug-induced thrombocytopenia can be related to binding of the IgG Fab

Received 29 August 2012 Returned for modification 9 September 2012 Accepted 23 September 2012

Published ahead of print 1 October 2012

Address correspondence to Charles Grégoire, charles.gregoire@gmail.com Copyright $\odot$ 2012, American Society for Microbiology. All Rights Reserved. doi:10.1128/AAC.01787-12 
fragment to circulating platelets. In our patient, enzyme-linked immunosorbent assays were negative for antibodies to platelet glycoproteins (anti Gp IIb/IIIa, anti Gp Ib/IX, and anti Gp Ia/IIa). This finding indicates either that the antibody recognized an untested glycoprotein target or that the drug acted as a hapten-eliciting antibody binding to the platelet surface (1). Finally, specific antibodies due to closely related chemicals can be present naturally, in the absence of previous drug exposure (1). Third, although the flow cytometry assay has been standardized for a wide range of drugs, the optimal plasma daptomycin concentration for antiplatelet antibody testing is not known. The use of an excessively high daptomycin concentration might result in nonspecific IgG binding to platelets. However, according to the model proposed by Bougie et al. (2), the drug concentration does not influence antibody binding. According to this model, a drug can react with both the antibody and the target protein, increasing the affinity of these two molecules for each other.

In conclusion, our case report strongly suggests that the re- cently introduced antibiotic daptomycin is associated with severe drug-dependent thrombocytopenia.

\section{ACKNOWLEDGMENT}

We thank A. Wolfe for helping to prepare the manuscript.

\section{REFERENCES}

1. Aster RH, Bougie DW. 2007. Drug-induced immune thrombocytopenia. N. Engl. J. Med. 357:580-587.

2. Bougie DW, Wilker PR, Aster RH. 2006. Patients with quinine-induced immune thrombocytopenia have both "drug-dependent" and "drugspecific" antibodies. Blood 108:922-927.

3. Carpenter CF, Chambers HF. 2004. Daptomycin: another novel agent for treating infections due to drug-resistant gram-positive pathogens. Clin. Infect. Dis. 38:994-1000.

4. Moore CL, et al. 2012. Daptomycin versus vancomycin for bloodstream infections due to methicillin-resistant Staphylococcus aureus with a high vancomycin minimum inhibitory concentration: a case-control study. Clin. Infect. Dis. 54:51-58.

5. Rousan TA, et al. 2010. Recurrent acute thrombocytopenia in the hospitalized patient: sepsis, DIC, HIT, or antibiotic-induced thrombocytopenia. Am. J. Hematol. 85:71-74. 